\title{
Vítimas de acidente motociclístico atendidas pelo serviço de atendimento móvel de urgência em Campo Grande, MS
}

Recebido em 04/04/2012

Aprovado em: 24/04/2013

Ahead of print: 16/05/2014
Sebastião Junior Henrique Duarte ${ }^{1}$ Renata Paiva Moret de Almeida Nardes²

Silvana Barbosa Pena ${ }^{3}$

Roberto Della Rosa Mendez ${ }^{4}$

Mariluci Camargo Ferreira da Silva Candido 5

Resumo: Objetivou-se descrever atendimentos de vítimas de acidentes motociclísticos atendidas pelo Serviço de Atendimento Móvel de Urgência em Campo Grande, MS. Estudo descritivo, realizado a partir da análise de 364 fichas de atendimento. Os resultados apontaram que a maioria das vítimas foram do sexo masculino, com 20 a 29 anos; membros inferiores/pelve e cabeça/pescoço foram as partes do corpo mais atingidas; maioria das vítimas foram encaminhadas para o hospital de referência ao trauma. Conclui-se que os acidentes motociclísticos elevam as taxas de morbidade e mortalidade por traumas, acarretam ônus social e o SAMU mostrou-se como um serviço de grande relevância.

Palavras-Chave: Motocicletas, Traumatismo múltiplo, Ferimentos e Lesões, Serviços Médicos de Emergência, Epidemiologia.

Victims of motorcycle accident attended by the mobile customer service urgency in Campo Grande, MS, Brazil

Abstract: This study aimed to describe the care of victims of motorcycle accidents attended by the Service Mobile Emergency Care in Campo Grande, MS. This descriptive study based on an analysis of 364 medical records. The results showed that most of the victims were male, 20 to 29 years; legs / pelvis and head / neck were the body parts most affected, most victims were admitted to the hospital in reference to trauma. We conclude that motorcycle accidents raise the rates of morbidity and mortality from trauma, lead burden and social SAMU showed up as a service of great importance.

Keywords: Motorcycles, Multiple Trauma, Wounds and Injuries, Emergency Medical Services, Epidemiology.

Víctimas de accidente de motocicleta que asistieron a la urgencia de los clientes de servicios móviles en Campo Grande, MS, Brasil

Resumen: Este estudio tuvo como objetivo describir la atención de las víctimas de accidentes de motocicleta que asistió el Servicio de Atención Móvil de Urgencia en Campo Grande, Brasil. Estudio descriptivo, basado en un análisis de 364 historias clínicas. Los resultados mostraron que la mayoría de las víctimas eran varones, de 20 a 29 años; piernas / pelvis y la cabeza / cuello fueron las partes del cuerpo más afectadas, la mayoría de las víctimas fueron ingresados en el hospital de referencia para trauma. Llegamos a la conclusión de que los accidentes de motocicleta aumentar las tasas de morbilidad y mortalidad por trauma, llevar carga y social SAMU se presentó como un servicio de gran importancia.

Palabras Clave: Motocicletas, Traumatismo Múltiple, Heridas y Traumatismos, Servicios Médicos de Urgencia, Epidemiología.

\section{INTRODUÇÃO}

$\mathrm{O}$ processo de urbanização está cada dia mais intenso, com isso se vive às consequências do crescimento desordenado das cidades e a presença da violência de modo geral ${ }^{(1)}$. Mesmo com as regras estabelecidas para organização da sociedade, nem todas as pessoas as conhecem e as cumprem como é o caso do código nacional de trânsito. $O$ cu mprimento da legislação do trânsito, certamente reduziria os acidentes dessa natureza, assim como os traumas ocasionados por eles, em especial os motociclísticos que contribuem para o aumento da mortalidade.

O trauma constitui um dos mais importantes e significativos problemas de toda a área social, enquanto a morte por afecções cardíacas ou câncer diminui em média 10 a 15 anos de vida de um cidadão, a decorrente do trauma chega a ceifar 30 a 40 anos de uma vida altamente produtiva, já que o problema incide prioritariamente sobre indivíduos jovens ${ }^{(2)}$.

Aproximadamente 60 milhões de pessoas sofrem algum tipo de traumatismo ao ano, contribuindo com uma em cada seis internações hospitalares. Esse fato demonstra que o trauma é um dos principais problemas de saúde pública em todos os países, independente do desenvolvimento sócio-econômico ${ }^{(3)}$.

Entre as vítimas mais vulneráveis dos acidentes de trânsito no Brasil estão os pedestres, os ciclistas e os motociclistas, estes representam mais de $50 \%$ dos mortos no trânsito. É evidente o aumento de acidentes de trânsito envolvendo motociclistas, principalmente devido à maior exposição do usuário nas vias públicas ${ }^{(4)}$, além da maior circulação deste veículo que vem ganhando, cada vez mais, a aceitação e a aprovação da população, pela agilidade e pelo custo reduzido, tanto para aquisição quanto para a manutenção.

O número de acidentes envolvendo moto é crescente e provoca trágicas consequencias devido a uma série de fatores, entre eles: 1) possibilitam uma maior exposição corpórea, 2) há maior dificuldade de visualização da moto por outros motoristas, 3) maior prevalência de comportamentos inadequados no trânsito(3).

${ }^{1}$ Enfermeiro. Doutor em Ciências da Saúde. Professor Adjunto da Universidade Federal de Mato Grosso do Sul (UFMS). Campus Três Lagoas, MS. E-mail:sihd@usp.br.

${ }^{2}$ Enfermeira. Especialista em Urgência e Emergência. Intervencionista do Serviço de Atendimento Móvel de Urgência de Campo Grande, MS.

${ }^{3}$ Enfermeira. Mestre. Professora Assistente da Universidade Federal de Mato Grosso do Sul. Campus Três Lagoas, MS.

${ }^{4}$ Enfermeiro. Doutor. Professor Adjunto da Universidade Federal de Mato Grosso do Sul (UFMS). Campus Três Lagoas, MS.

${ }^{5}$ Enfermeira. Doutora em Enfermagem Psiquiátrica. Professora Adjunta do Mestrado em Enfermagem e da Graduação em Enfermagem da Universidade Federal de Mato 
Desse modo, parte-se do pressuposto de que o conhecimento dos diferentes tipos de acidentes e suas vítimas contribui para a definição de políticas de prevenção e controle desses agravos e das mortes por eles causadas, especialmente os acidentes motociclisticos, ainda, a educação em saúde que promova a consciência cidadã e a prevenção de danos como as amputações ${ }^{(5)}$ e até mesmo a morte.

Tomando a relevância da temática é que o estudo objetivou descrever os atendimentos prestados às vítimas de acidentes motociclísticos atendidas pelo Serviço de Atendimento Móvel de Urgência em Campo Grande, MS, considerando que o primeiro passo para o estudo de um agravo é a descrição exata e minuciosa de como ele acontece na população.

\section{METODOLOGIA}

Trata-se de um estudo descritivo, exploratório, retrospectivo, de abordagem quantitativa que utilizou dados fornecidos pelo Serviço de Atendimento Móvel de Urgência (SAMU) do município de Campo Grande, MS, referente ao período de abril de 2005 a abril de 2007, correspondente a seus dois primeiros anos de operação.

Campo Grande é a capital do Estado de Mato Grosso do Sul, com população de cerca de 900 mil habitantes. Conta com o SAMU desde abril de 2005.

A pesquisa foi realizada na Central de Regulação de Urgências do SAMU, onde ficam arquivados os registros das ocorrências. As fichas de atendimento foram preenchidas pelos profissionais que prestaram o atendimento.

A população foi constituída pelas vítimas de acidentes motociclísticos, atendidas pelas unidades de suporte avançado no período estudado.

Foram selecionadas somente as ocorrências atendidas pelo suporte avançado, que totalizaram 364 prontuários, constituindo 402 pessoas atendidas, considerando que algumas ocorrências envolviam até duas pessoas em cada motocicleta.

Os dados foram coletados de fevereiro a junho de 2008 na sede administrativa do SAMU, utilizando-se das fichas de atendimentos (prontuários), manipulados pelos pesquisadores.

As variáveis eleitas para o estudo foram as que constavam no prontuário, categorizadas em dados de identificação da vítima (sexo e idade), região anatômica atingida, localização geográfica em que a vítima se encontrava (urbana, rural ou outra), distrito do município em que o indivíduo foi acometido pelo acidente motociclístico e local de destino do encaminhamento.

Os dados foram inicialmente dispostos em planilha elaborada para esse fim, sem necessidade de pré-teste, considerando-se que o prontuário do SAMU é padronizado. Na análise, utilizou-se o programa estatístico SPSS, versão 15.0, para cálculo de frequências e porcentagens.

Os resultados foram organizados na forma de estatística descritiva e apresentados em tabelas.

O projeto de pesquisa foi aprovado pelo Comitê de Ética em Pesquisa Envolvendo Seres Humanos da Universidade Federal de Mato Grosso do Sul (protocolo 1.153/2008). Foi assinado termo de compromisso para utilização de informações de banco de dados, em atendimento à Resolução 196/96 do Conselho Nacional de Saúde(6).

\section{RESULTADOS}

Nos dois primeiros anos de implantação do SAMU em Campo Grande (abril de 2005 a abril de 2007), as unidades de suporte avançado de vida (USA) foram acionadas para atender aproximadamente 7.055 ocorrências. Destas 364 foram por trauma motociclísticos, representando 5\% dos casos, totalizando 402 pessoas, pois em algumas ocorrências havia duas pessoas na moto. Uma média de 3.528 atendimentos por ano e 294 atendimentos por mês(7).

Com relação a caracterização dos sujeitos a Tabela 1 mostra a distribuição das vítimas por sexo e faixa etária.

Tabela 1 - Distribuição quanto às características demográficas sexo e faixa etária das vítimas de acidente motociclístico, atendidos pelo SAMU/Campo Grande, MS, abr 2005 a abr 2007.

\begin{tabular}{|c|c|c|}
\hline Sexo & $\mathbf{n}$ & $\%$ \\
\hline Masculino & 316 & 79 \\
\hline Feminino & 86 & 21 \\
\hline Total & $\mathbf{4 0 2}$ & $\mathbf{1 0 0}$ \\
\hline (em anos) & $\mathbf{n}$ & $\%$ \\
\hline 0 a 10 & 14 & 3 \\
\hline 11 a 19 & 59 & 15 \\
\hline 20 a 29 & 178 & 44 \\
\hline 30 a 39 & 79 & 100 \\
\hline 40 a 49 & 46 & 11 \\
\hline 50 a 59 & 18 & 5 \\
\hline Total & 802 & 20 \\
\hline
\end{tabular}

As lesões decorrentes dos eventos traumáticos resultam em deficiências e incapacidades temporárias ou permanentes, que interferem na capacidade das vítimas sobreviventes cumprirem tarefas que delas são esperadas, assim como na qualidade de suas vidas. Nesse sentido a Tabela 2 aponta às regiões anatômicas mais atingidas.

Tabela 2 - Distribuição quanto à região anatômica das vítimas de acidente motociclístico atendidos pelo SAMU/Campo Grande, MS, abril/2005 a abril/2007.

\begin{tabular}{|c|c|c|}
\hline $\begin{array}{c}\text { Regiöes anatômicas } \\
\text { mais atingidas }\end{array}$ & $\mathbf{n}$ & $\%$ \\
\hline $\begin{array}{c}\text { Membros inferiores/ } \\
\text { pelve }\end{array}$ & 158 & 39 \\
\hline Cabeça e pescoço & 112 & 28 \\
\hline Membros superiores & 88 & 22 \\
\hline
\end{tabular}




\begin{tabular}{|c|c|c|}
\hline Tórax & 32 & 8 \\
\hline Dorso & 8 & 2 \\
\hline Abdome & 4 & 1 \\
\hline Total & $\mathbf{4 0 2}$ & 100 \\
\hline
\end{tabular}

A localidade das ocorrências é apresentada na Tabela 3 e ilustra a distribuição entre a zona urbana, rural e rodovias.

Tabela 3 - Distribuição quanto a região de ocorrência das vítimas atendidas de acidente motociclístico atendidos pelo SAMU/Campo Grande, MS, abril/2005 a abril/2007.

\begin{tabular}{|l|c|c|}
\hline Regiăo & $\mathbf{n}$ & $\%$ \\
\hline Urbana & 382 & 95 \\
Rural & 4 & 1 \\
\hline Rodovias & 16 & 4 \\
\hline Total & $\mathbf{4 0 2}$ & $\mathbf{1 0 0}$ \\
\hline
\end{tabular}

Com relação ao distrito urbano da cidade de Campo Grande onde concentraram maior número de vítimas atendidas a Tabela 4 aponta tais localidades.

Tabela 4 - Distribuição quanto ao distrito de ocorrência das vítimas de acidente motociclístico, atendidos pelo SAMU/ Campo Grande, MS, abril/2005 a abril/2007.

\begin{tabular}{|l|c|c|}
\hline Distrito & $\mathbf{n}$ & \% \\
\hline Sul & 165 & 41 \\
\hline Leste & 137 & 34 \\
Norte & 64 & 16 \\
Oeste & 36 & 9 \\
\hline Total & $\mathbf{4 0 2}$ & $\mathbf{1 0 0}$ \\
\hline
\end{tabular}

Os dados mostram que houve predomínio das ocorrências nas regiões Sul e Leste do município com 165 (41\%) e 137 (34\%) vítimas. Ressalta-se que a região Sul de Campo Grande, MS, concentra o maior número de pessoas da classe sócioeconômica baixa e média, enquanto na região Leste há grande parte do setor comercial do município.

Quanto ao destino das vítimas a Tabela 5 mostra que as equipes conduziram para diversas localidades.

Tabela 5 - Distribuição quanto ao local de destino das vítimas de acidente motociclístico, atendidos pelo SAMU/Campo Grande, MS, abril/2005 a abril/2007.

\begin{tabular}{|c|c|c|}
\hline Local & $\mathbf{n}$ & $\%$ \\
\hline $\begin{array}{c}\text { Santa casa } \\
\text { Centro Regional } \\
\text { de Saúde }\end{array}$ & 250 & 62 \\
$\begin{array}{c}\text { Hospital } \\
\text { Universitário }\end{array}$ & 80 & 20 \\
\hline
\end{tabular}

\begin{tabular}{|c|c|c|}
\hline Liberação no local & 12 & 3 \\
\hline Hospital Regional & 8 & 2 \\
Óbito no local & 8 & 2 \\
\hline Hospital Militar & 3 & 0.7 \\
$\begin{array}{c}\text { Centro } \\
\text { Ortopédico }\end{array}$ & 1 & 0.3 \\
\hline Total & $\mathbf{4 0 2}$ & $\mathbf{1 0 0}$ \\
\hline
\end{tabular}

Observa-se que 62\% das vítimas foram levadas para a Santa Casa. Esse hospital é referência no atendimento à pacientes com grandes traumas, principalmente nas especialidades de cirurgias neurológicas, ortopédicas, pediátricas e gerais, para todo o Estado de Mato Grosso do Sul.

Para o Hospital Universitário e Hospital Regional são destinados casos ortopédicos isolados e traumas abdominais. Esses hospitais atendem prioritariamente casos clínicos e cirúrgicos, embora haja atendimento em outras especialidades.

No município de Campo Grande, MS, existem 6 Centros Regionais de Saúde com atendimento 24 horas e 3 Unidades de Pronto Atendimento à Saúde (UPAS). Nas UPAS é possível manter o paciente em observação por mais de 12 horas. Conta-se com aparelho de ultrassonografia e raio- $X$, inclusive respirador artificial. O laboratório municipal também tem atendimento 24 horas, contribuindo para o atendimento integral e resolutivo.

Das ocorrências realizadas pelas unidades de suporte avançado doSAMU,em queas vítimas nãoforam transportadas, embora tenham recebido atendimento, somaram 12 vítimas (3\%) que tiveram apenas escoriações e foram liberadas no local do atendimento (via pública); 8 vítimas (2\%) sofreram lesões graves, incompatíveis com a vida vindo a óbito no local, ficando aos cuidados da equipe da polícia.

\section{DISCUSSÃO}

Os dados mostram que as vítimas de trauma motociclísticos foram 316 (79\%) do sexo masculino e 86 (21\%) do sexo feminino, uma relação de 3 homens para 1 mulher. Esses resultados são semelhantes a outros estudos nacionais que apontam para a predominância do sexo masculino entre as vítimas de acidentes de trânsito ${ }^{(3,8)}$.

Observa-se que a faixa etária mais acometida pelos acidentes motociclístico esteve entre 20 a 29 anos de idade (44\% dos casos). As unidades de suporte avançado do SAMU atenderam, portanto, população jovem, economicamente ativa. Em algumas situações a opção por locomover-se de moto, se dá devido ao menor custo para aquisição e manutenção.

No Brasil os acidentes de trânsito têm merecido políticas que visam reduzir o trauma, as sequelas decorrentes e o impactos sociais, econômicos e pessoais que deles derivam(5). Os dados desse estudo estão em consonância com a população em geral, onde os adultos jovens foram os mais atingidos pelos acidentes de trânsito, requerendo medidas de prevenção do trauma nesse segmento populacional.

Ficou evidente que em Campo Grande, MS, um dos fatores de morbidade é o aumento no número de acidentes envolvendo motocicletas, visto que esse meio de transporte vem ganhando a aceitação e a aprovação pela população, visto ser um veículo ágil, econômico e de custo reduzido ${ }^{(4)}$. 
No entanto a situação é preocupante e merece atenção por parte de vários segmentos, considerando que $\mathrm{O}$ desenvolvimento da nação depende, entre outros fatores relevantes, da força de trabalho e do nível educacional das pessoas. Acidentes nessa faixa etária gera ônus para a sociedade tanto no campo da assistência à saúde (atendimento pré-hospitalar, hospitalar e pós-hospitalar) quanto previdenciário (benefícios por motivo de doença ou aposentadorias precoces).

Observa-se um predomínio das lesões em membros inferiores/pelve, ocorrendo em 158 casos (39\%), seguidos por 112 lesões (28\%) em cabeça/pescoço; 88 (22\%) nos membros superiores. Os dados chamam atenção para o potencial de complicações e, até, letalidade, pois trauma em estruturas ósseas podem provocar graves hemorragias como é o caso de fraturas em membros e pelve. Outro alerta que o estudo aponta são os traumas em cabeça e pescoço, podendo levar ao traumatismo crâniano e raquimedular, requerendo atendimento de especialidades raras pelo Sistema Único de Saúde; no entanto o primeiro atendimento prestado pelo SAMU mostrou-se fundamental para preservar a vida da maioria dos socorridos, considerando que para 8 vítimas (2\%) houve incompatibilidade com a vida evoluindo para óbito no local.

Ressalta-se que a vulnerabilidade do motociclista é evidente. Na colisão, que é um dos tipos de acidentes de motocicleta mais usual, o motociclista absorve em sua superfície corpórea toda a energia gerada no impacto, seja de encontro com a via pública, ou com os objetos da mesma e até outros veículos a motor ${ }^{(4)}$.

Em Catanduva- $\mathrm{SP}^{(3)}$, os resultados foram muito semelhantes havendo predomínio das lesões em membros inferiores/pelve e cabeça/pescoço. As lesões em membros inferiores se devem ao fato da moto sofrer impacto frontal ou lateral. No primeiro caso, a moto inclina-se para frente projetando o motociclista contra o guidom. Caso os pés e as pernas continuem fixas no pedal, a coxa será lançada contra o guidom podendo haver lesões bilaterais dos membros inferiores. Na segunda situação as lesões ocorrem devido a compressão das pernas e cintura pélvica contra o anteparo ou outro veículo ${ }^{(3)}$. Provavelmente as vítimas atendidas pelo SAMU de Campo Grande, MS, tenham sofrido situação semelhante.

Das lesões ocorridas na região de cabeça/pescoço, 44 (39\%) causaram traumas leves; 9 (8\%) foram traumas moderados e 14 (13\%) traumas graves. Houve 39 (35\%) lesões em face, associadas ao uso do capacete aberto. Em 6 casos (5\%) as lesões foram na região do pescoço. Esses dados corroboram com os benefícios do uso do capacete, evidente na gravidade do trauma que mostrou-se de leve a moderado, em contrapartida o mal uso do capacete levou a ferimentos na face.

Em $85 \%$ das vítimas as lesões em tórax e abdome estavam relacionadas a gravidade, provocando alterações respiratórias e/ou sinais de choque hipovolêmico. A intervenção da equipe que compõe o suporte avançado de vida propicia as chances de preservação da vida das vítimas, além da redução de danos relacionados à assistência.

As anotações nas fichas de atendimentos das vítimas revelaram que as lesões em membros inferiores e pelve estiveram relacionadas a fraturas, luxações, amputação, lesão vascular, déficit motor e contusões. Outra situação em que a equipe do SAMU pôde atuar na prevenção de complicações decorrentes de trauma nessas estruturas corporais, evitando a morte. Vale destacar que a equipe multiprofissional que integra a ambulância de suporte avançado conta com materiais e equipamentos que permitem intervenções necessárias para evitar complicações como o choque hipovolêmico, hemorragias, parada cardiorespiratória, entre outras, com isso a manutenção da vida até a chegada ao hospital.

Em relação à região geográfica de ocorrência, a maioria, 382 (95\%) casos de traumas motociclísticos ocorreram no perímetro urbano. Estima-se que o predomínio das ocorrências de traumas na região urbana aconteça pela maior concentração de veículos nesta área e o acionamento do SAMU é mais frequente por populares que transitam os locais onde tenha ocorrido o acidente, na tentativa de socorro às vítimas.

O destino das vítimas é relevante uma vez que mais importante do que levar a vítima de trauma para o hospital mais próximo é levar a vítima com segurança para o hospital adequado, ou seja, para o hospital que possua infra-estrutura e pessoal habilitado para atendimento às necessidades da vítima $^{(8,9)}$

\section{CONCLUSÃO}

A limitação do estudo está na população estudada, considerando que foram pesquisados os atendimentos realizados pelas unidades de suporte avançado (USA), nos dois primeiros anos de implantação do serviço de atendimento móvel de urgência (SAMU). O estudo não esgota os atendimentos realizados pelo (SAMU) de Campo Grande, MS, pois foram analisados somente os acidentes motociclísticos atendidos pelas equipes de suporte avançado de vida, ou seja, envolvendo os traumas mais graves ou com potencial para agravamento, não sendo computados os atendimentos feitos pelas Unidades de Suporte Básico (USB) e nem os transportes secundários, constituindo motivação para novas pesquisas.

A descrição dos atendimentos revelou que as vítimas mais acometidas são do sexo masculino e na faixa de idade entre 20 a 29 anos, ou seja, indivíduos economicamente ativos que deixam de contribuir e passam a gerar ônus para a sociedade. Ficou evidente que o SAMU, como integrante das redes de atenção à saúde, destinou às vítimas para os locais onde ofereceram a continuidade do cuidado, dentro do nível de complexidade requerida, como é o caso da Santa Casa de Campo Grande, MS, hospital referência para atendimento a politrauma e neurologia, além de outras especialidades, possuindo infraestrutura e pessoal especializado para atendimento às diversas necessidades das vítimas.

Apesar de algumas limitações, acredita-se que este estudo conseguiu traçar o perfil das vítimas estudadas, fornecendo informações importantes sobre os grupos que mais sofreram acidentes motociclísticos o que poderá oferecer subsídios para ações preventivas e de controle, em relação tanto à morbidade quanto à mortalidade dessas. 


\section{Referências}

1. Sanches S, Duarte SJH, Pontes ERJC. Caracterização das vítimas de ferimentos por arma de fogo, atendidas pelo Serviço de Atendimento Móvel de Urgência em Campo Grande-MS. Saúde soc. 2009; 18(1):95102. Disponível em: http://www.scielo.br/pdf/sausoc/v18n1/en_10.pdf 2. Chavaglia SRR, Amaral SEM, Bittar DB, Ferreira PM, Barbosa MH. Vítimas de trauma por causas externas na cidade de Uberaba - MG. Mundo saúde. 2008; 32(1): 100-106. Disponível em: http://www. saocamilo-sp.br/pdf/mundo_saude/58/100a106.pdf

3. Batista SEA, Baccani RJA, Silva RAP, Gualda KPF, Vianna Júnior RJA Análise comparativa entre os mecanismos de trauma, as lesões e o perfil de gravidade das vítimas em Catanduva - SP. Rev Col Bras Cir. 2006; 33(1): 6-10. Disponível em: http://www.scielo.br/pdf/rcbc/v33n1/ v33n1a02.pdf

4. Sado MJ, Morais FD, Viana, FD. Caracterização das vítimas por acidentes motociclísticos internados no hospital de urgência de Goiânia. Rev Movim. 2009; 2(2): 49-53. Disponível em: http://www.nee.ueg.br/ seer/index.php/movimenta/article/viewFile/248/202

5. Montiel A, Vargas MAO, Leal SMC. Caracterização de pessoas submetidas à amputação. Enferm Foco. 2012; 3(4):169-73. Disponível em: http://revista.portalcofen.gov.br/index.php/enfermagem/article/ view/377/168

6. Ministério da Saúde; Conselho Nacional de Saúde. Resolução № 196/96 Normas regulamentadoras de pesquisa envolvendo seres humanos. Brasília (Brasil): Ministério da Saúde; 1996.

7. Serviço de Atendimento Móvel de Urgência. Relatório Anual do serviço. Campo Grande: SAMU. 2007.

8. Duarte SJH, Lucena BB, Morita LHM. Atendimentos prestados pelo Serviço Móvel de Urgência em Cuiabá, MT, Brasil. Rev.Eletr. Enf. 2011; 13(3): 502-07. Disponível em: http://www.fen.ufg.br/fen_revista/v13/n3/pdf/v13n3a16.pdf 9. Canova JCM, Bueno MFR, Oliver CCD, Souza LA, Belati LA, Cesarino CB, et al. Traumatismo cranioencefálico de pacientes vítimas de acidentes de motocicletas. Arq Ciênc Saúde. 2010; 17(1): 9-14. Disponível em: http://www. cienciasdasaude.famerp.br/racs_ol/vol-17-1/IDL_jan-mar_2010.pdf 